\title{
Sensitivity of C-Tb: a novel RD-1-specific skin test for the diagnosis of tuberculosis infection
}

\author{
Soren T. Hoff ${ }^{2}$, Jonathan G. Peter ${ }^{1}$, Grant Theron ${ }^{1}$, Mellissa Pascoe ${ }^{1}$, \\ Pernille N. Tingskov ${ }^{3}$, Henrik Aggerbeck $^{3}$, Daniel Kolbus ${ }^{3}$, Morten Ruhwald $^{2}$, \\ Peter Andersen ${ }^{2,4}$ and Keertan Dheda ${ }^{1,4}$
}

Affiliations: ${ }^{1}$ Lung Infection and Immunity Unit, Division of Pulmonology and UCT Lung Institute, Dept of Medicine, University of Cape Town, Cape Town, South Africa. ${ }^{2}$ Dept of Infectious Diseases Immunology, Statens Serum Institut, Copenhagen, Denmark. ${ }^{3}$ Dept of Vaccine Development, Statens Serum Institut, Copenhagen, Denmark. ${ }^{4}$ Joint senior authors.

Correspondence: Keertan Dheda, H47 Old Main Building, Groote Schuur Hospital, Observatory 7925, South Africa. E-mail: keertan.dhedaduct.ac.za

ABSTRACT C-Tb, a novel Mycobacterium tuberculosis and 6-kDa early secretory antigenic target/10-kDa culture filtrate protein (ESAT-6/CFP-10)-specific skin test, has high specificity in bacille Calmette-Guerinvaccinated healthy controls. However, the sensitivity of $\mathrm{C}$ - $\mathrm{Tb}$ has hitherto not been determined. The objective was to determine the sensitivity of $\mathrm{C}$ - $\mathrm{Tb}$ in patients with active tuberculosis $(\mathrm{TB})$ in comparison with the tuberculin skin test (TST) and QuantiFERON-TB Gold In-Tube (QFT-GIT).

$\mathrm{C}-\mathrm{Tb}$ and TST were randomly administered in a double-blinded fashion to one or the other forearm in 253 patients with active TB with or without HIV co-infection. QFT-GIT testing was performed prior to skin testing.

Using a receiver operating characteristic curve-derived cut-point of $5 \mathrm{~mm}, \mathrm{C}$-Tb sensitivity was similar to QFT-GIT (73.9 (95\% CI 67.8-79.3) versus 75.1 (95\% CI 69.3-80.2)), and similar in HIV-infected and HIV-uninfected patients (76.7 (95\% CI 69.0-83.3) versus 69.5 (95\% CI 59.2-78.5)). However, sensitivity was significantly diminished in HIV-infected patients with CD4 counts $<100$ cells $\cdot \mathrm{mm}^{-3}$. C-Tb and QFTGIT combined had significantly higher sensitivity than $\mathrm{C}$-Tb alone $(\mathrm{p}<0.0001)$. C-Tb was safe with no significant adverse events. The $5 \mathrm{~mm}$ cut-point corresponded to that found in the previously published specificity study (TESEC-04).

C-Tb has similar sensitivity compared with QFT-GIT for the diagnosis of M. tuberculosis infection. Sensitivity was reduced only in HIV-infected patients with severe immunosuppression. Further studies in different settings are required to validate the proposed $5 \mathrm{~mm}$ cut-point.

@ERSpublications

C-Tb has similar sensitivity compared with QFT-GIT for the diagnosis of $M$. tuberculosis infection http://ow.ly/TtFf6

Editorial comment in: Eur Respir J 2016; 47: 704-706 [DOI 10.1183/13993003.01910-2015].

This article has supplementary material available from erj.ersjournals.com

Received: Sept 032015 | Accepted after revision: Oct 092015 | First published online: Dec 172015

Clinical trial: This study is registered at www.clinicaltrials.gov with identifier number NCT01241188.

Support statement: The trial was funded by Statens Serum Institut. Funding information for this article has been deposited with FundRef.

Conflict of interest: Disclosures can be found alongside the online version of this article at erj.ersjournals.com

Copyright OERS 2016 


\section{Introduction}

Unlike those with active tuberculosis (TB) disease, individuals with latent TB infection (LTBI) are asymptomatic, without radiological signs of active disease, and it is not possible to microbiologically detect bacteria in the sputum or other biological samples. Detection and treatment of LTBI is important for the complete eradication of TB $[1,2]$. The only way to currently diagnose presumed LTBI is by detecting the host immune response to Mycobacterium tuberculosis-derived antigens and thus indirectly inferring the prior or current presence of mycobacteria [3,4]. This approach has been used to infer the presence of LTBI using the purified protein derivative (PPD)-related tuberculin skin test (TST). PPD TST is easy to use, but false-positive reactions may occur in individuals vaccinated with bacille Calmette-Guerin (BCG) vaccine, particularly in infants after birth, or in individuals infected with nontuberculous mycobacteria [5].

To address this drawback, two commercially available in vitro immunodiagnostic assays (QuantiFERON-TB Gold In-Tube (QFT-GIT; Cellestis/Qiagen, Venlo, the Netherlands) and T-Spot.TB (Oxford Immunotec, Abingdon, UK)) have been developed. They interrogate effector T-cell responses to two immunogenic M. tuberculosis antigens, i.e. $6-\mathrm{kDa}$ early secretory antigenic target (ESTA-6) and $10-\mathrm{kDa}$ culture filtrate protein (CFP-10), which are present in only a few mycobacteria apart from $M$. tuberculosis (e.g. Mycobacterium marinum, Mycobacterium kansasii, etc.), and are not found in any of the BCG vaccine strains used worldwide [6-9]. However, these tests are expensive, require phlebotomy (technically difficult in children and some adults), transport of samples to a laboratory with availability of required equipment and infrastructure (e.g. ELISPOT and ELISA readers), and reporting of the results back to peripheral healthcare centres. Both tests have been shown to have high reversion rates [10].

Simpler and more-user-friendly tests are therefore required. This unmet need has now been addressed with the development of C-Tb, a novel M. tuberculosis-specific skin test that contains ESAT- 6 and CFP-10 antigens [11-13]. Alternative versions have been made in China and Russia [14, 15]. The C-Tb skin test can be used by health services to test for M. tuberculosis infection and is applied in exactly the same way as the TST, but without the suboptimal specificity. Two prior clinical trials found C-Tb to be safe and well tolerated in both healthy subjects and in patients with active TB [12, 13] (TESEC-01, -02 and -03). In order to identify the optimal cut-point for C-Tb, a specificity trial was conducted in 151 presumed uninfected and BCG-vaccinated healthy adults from the UK. This confirmed the superior specificity of C-Tb compared with TST [13].

Here, we report on the results from the sensitivity trial in TB patients with active TB that served as a surrogate for prior contact with $M$. tuberculosis. The primary objective was to provide sensitivity data for $\mathrm{C}-\mathrm{Tb}$, which in combination with previously published specificity data would allow the derivation of an optimal cut-point for C-Tb.

\section{Materials and methods}

Trial design

Eligible participants were HIV-infected or HIV-uninfected adults aged 18-65 years with a recent diagnosis of active TB. The trial was conducted from April to December 2011 at Langa, Gugulethu, Chapel Street, Du Noon, Table View, Nyanga, Sea Winds, Lotus River, Phillipi, Retreat and Manenberg clinics in Cape Town, South Africa. At visit 1, participants were assessed for eligibility, informed consent was obtained, and medical examinations including blood sampling for safety laboratory tests and QFT-GIT were performed. All participants received C- Tb $0.1 \mathrm{~mL}(0.1 \mu \mathrm{g})$ in one arm and TST $0.1 \mathrm{~mL}$ (2 tuberculin units (TU) tuberculin PPD RT 23 SSI) in their other arm in a double-blinded manner. The arm used for each intervention was randomised. Induration reactions at the injection sites were read after 2-3 days (visit 2) and recorded in millimetres $(\mathrm{mm})$. The absence of induration was recorded as $0 \mathrm{~mm}$. The last follow-up (visit 3) was scheduled at day 28, and included a medical examination and blood sampling for safety laboratory tests. Recruitment and arm-specific randomisation was in keeping with the CONSORT guidelines [16].

\section{Ethical approvals}

The trial was sponsored by Statens Serum Institut (www.clinicaltrials.gov: NCT01241188; TESEC-04), and approved by the Faculty of Health Sciences Human Research Ethics Committee, Cape Town, South Africa (reference number 515/2010) and by the Medicines Control Council, South Africa. The trial was conducted in accordance with the principles of Good Clinical Practice and the World Medical Association (WMA) Declaration of Helsinki adopted by the 18th WMA General Assembly, Helsinki, Finland, 1964 and subsequent amendments.

\section{Safety assessment}

Adverse events were assessed and recorded in diaries in the first hour after injection and at the follow-up visits by the trial staff. All adverse events were assessed for intensity, relatedness to skin testing, outcome 
and seriousness as stated in the trial protocol. Safety laboratory tests that were out of normal ranges used at the trial site were recorded as adverse events. All adverse events were coded according to the investigator's original description to the Lowest Level Term with Preferred Term and System Organ Class using MedDRA version 14.1 (www.meddra.org). A Data Safety Monitoring Board was established consisting of three independent senior clinicians, with the function to give expert advice to the Principal Investigator on issues regarding safety of the participants.

\section{C-Tb and PPD TST}

$\mathrm{C}-\mathrm{Tb}$ was manufactured by Statens Serum Institut (Copenhagen, Denmark) according to Good Manufacturing Practice standards as a solution of recombinant ESAT-6 and CFP-10 mixed in a weight ratio of 1:1. Both antigens were cloned and expressed in Lactococcus lactis [17]. Tuberculin PPD RT 23 SSI $2 \mathrm{TU} / 0.1 \mathrm{~mL}$ (Statens Serum Institut) was used for PPD TST. Both C-Tb and PPD TST were injected intradermally by the Mantoux technique as previously described [12, 17]. PPD TST responses were evaluated at cut-points of $\geqslant 5, \geqslant 10$ and $\geqslant 15 \mathrm{~mm}$.

\section{QFT-GIT test}

QFT-GIT tests were performed and analysed according the manufacturer's instructions. Blood sampling for the QFT-GIT tests was done at visit 1 prior to administration of C-Tb and PPD TST.

\section{Statistics}

Statistical analyses were performed using SAS JMP (www.jmp.com), or Prism 6 or GraphPad online statistical software (www.graphpad.com).

\section{Results}

\section{Trial population}

Eligible participants were HIV-infected or HIV-uninfected adults aged 18-65 years with a recent diagnosis of active TB. 258 volunteers were screened and 253 participants were included in the trial (figure 1). Reasons for noneligibility were use of prednisolone, TB treatment for $>2$ weeks, TB relapse and breastfeeding. Of the 253 included, 153 were HIV-uninfected and 100 were HIV-infected.

All HIV-uninfected participants were on TB treatment for recently diagnosed active TB confirmed by at least one positive sputum smear microscopy or positive culture result. All HIV-infected participants (HIV infection confirmed by two positive rapid tests or one positive rapid test plus by positive Enzygnost anti-HIV-1/2 Plus ELISA) were on TB treatment for recently diagnosed active TB with a clinical picture of TB according to South African guidelines. No participants had received TB treatment for $>2$ weeks at inclusion. All 253 participants were included in the safety analyses. One participant withdrew from the trial before the second visit. A total of 241 participants (95 HIV-infected and $146 \mathrm{HIV}$-uninfected) were included in the per protocol population which was used for immune response analyses. 11 participants were excluded from the per protocol population due to major protocol deviations.

\section{Demographics}

The majority of baseline characteristics were similar for HIV-uninfected and HIV-infected patients with the exceptions that the HIV-infected population included a higher proportion of females, individuals of African descent and negative sputum smear microscopy results (table 1). There were no significant correlations between $\mathrm{C}-\mathrm{Tb}$ induration size versus age or sex (logistic regression, data not shown).

\section{Magnitude of C-Tb, TST and QFT-GIT responses}

The median C- $\mathrm{Tb}$ response was $23 \mathrm{~mm}$ (95\% CI 20-25; interquartile range (IQR) 0-34) in all participants, $23 \mathrm{~mm}$ (95\% CI 20-26; IQR 10-33) in HIV-uninfected and $22 \mathrm{~mm}$ (95\% CI 18-25; IQR 0-34) in HIV-infected (figure 2a-c). The median TST response was $25 \mathrm{~mm}$ (95\% CI: 23-26; IQR 19-30) in all participants, $25 \mathrm{~mm}$ (95\% CI 24-28; IQR 21-31) in HIV-uninfected and $24 \mathrm{~mm}$ (95\% CI 19-25; IQR $15-29)$ in HIV-infected (figure $2 \mathrm{~d}$ and $\mathrm{f}$ ). There was no significant difference $(\mathrm{p}=0.3452$ and $\mathrm{p}=0.1348$ ) between HIV-infected and HIV-uninfected distributions for C-Tb or TST. QFT-GIT median responses were $2.3 \mathrm{IU} \cdot \mathrm{mL}^{-1}$ for both $\mathrm{HIV}$-uninfected and HIV-infected (online supplementary figure S5). C-Tb responses correlated positively with TST (Spearman rank $r=0.6442$ in HIV-uninfected and $r=0.7253$ in HIV-infected; $\mathrm{p}<0.0001$ for both) and with QFT-GIT responses ( $\mathrm{r}=0.4897$ in HIV-uninfected and $\mathrm{r}=0.5977$ in HIV-infected; $\mathrm{p}<0.0001$ for both).

\section{C-Tb cut-point analysis}

A cut-point for a positive $\mathrm{C}-\mathrm{Tb}$ test was determined using receiver operating characteristic (ROC) curve analysis comparing $\mathrm{C}$ - $\mathrm{Tb}$ induration responses from the $146 \mathrm{HIV}$-uninfected participants with confirmed 


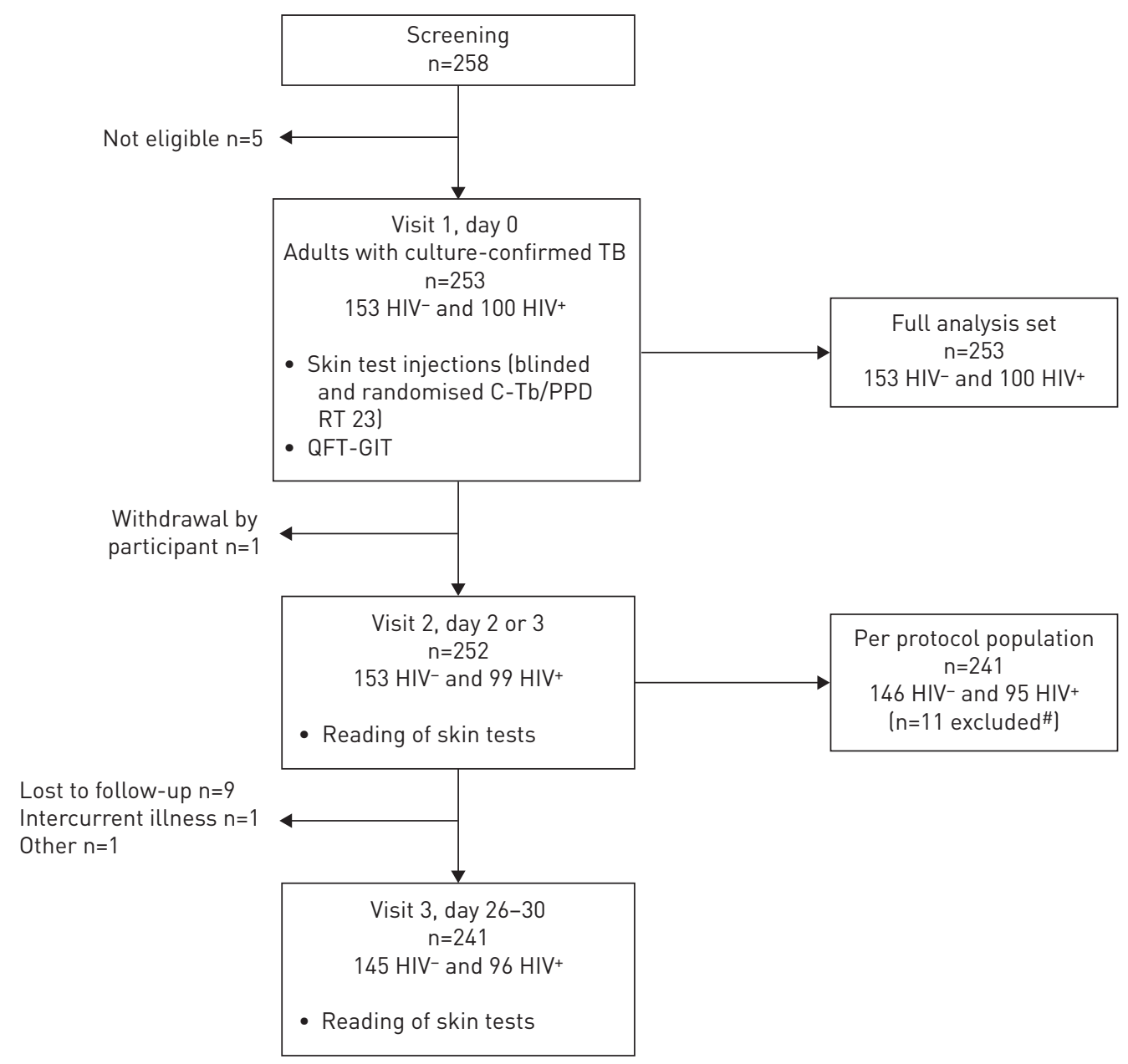

FIGURE 1 Flow diagram of the trial procedures and trial population. TB: tuberculosis; QFT-GIT: QuantiFERON-TB Gold In-Tube. \#: reasons for exclusion from per protocol population were major protocol deviations, and included injection failures $(n=2)$, unconfirmed TB diagnosis $(n=6)$, both injection failure and unconfirmed TB diagnosis ( $n=1)$, use of immune-suppressive drug $(n=1)$ or second visit out of visit window $(n=1)$.

TB and 147 BCG-vaccinated healthy controls from the previously published specificity trial (data shown in online supplementary figure S6) [13]. The area under the curve was 0.88 and suggested $\geqslant 5 \mathrm{~mm}$ as the optimal cut-off for $\mathrm{C}-\mathrm{Tb}$ (figure 3). Subsequent inclusion of the HIV-infected participants into the analysis gave the same result (not shown). The $\mathrm{C}-\mathrm{Tb} \geqslant 5 \mathrm{~mm}$ cut-point was used in all subsequent analyses. C-Tb specificity at $\geqslant 5 \mathrm{~mm}$ was $99.3 \%$ (95\% CI 96.0-100) as reported by AGGERBECK et al. [13].

\section{Sensitivity of C-Tb, TST and QFT-GIT}

Overall C-Tb sensitivity was 73.9\% (95\% CI 67.8-79.3), and similar in HIV-infected and HIV-uninfected participants (76.7\% (95\% CI 69.0-83.3) versus 69.5\% (95\% CI 59.2-78.5); $\mathrm{p}=0.232$ ). C-Tb and QFT-GIT showed comparable rates of positive test results (73.9\% versus $75.1 \%)$ in all participants grouped, as well as in HIV-uninfected and HIV-infected. TST showed higher sensitivity than both C-Tb and QFT-GIT in all participants as well as when split between HIV-uninfected and HIV-infected (table 2). 19 participants (7.9\%) had indeterminate QFT-GIT results (13 HIV-uninfected and six HIV-infected; online supplementary table S5). If QFT-GIT indeterminate results were excluded from analysis, QFT-GIT sensitivity was higher than $\mathrm{C}-\mathrm{Tb}(\mathrm{p}=0.03)$.

\section{Discordant results}

Excluding participants with a QFT-GIT indeterminate result, C-Tb and QFT-GIT agreed on 78\% of all test results, on $75 \%$ in HIV-uninfected and on $83 \%$ in HIV-infected (online supplementary table S6). The two tests were significantly different in McNemar's test for marginal homogeneity $(\mathrm{p}=0.030)$, but not when split between HIV-uninfected and HIV-infected ( $\mathrm{p}=0.164$ and $\mathrm{p}=0.121)$. In contrast, for $\mathrm{C}$-Tb versus TST, McNemar's test was significant in all populations regardless of whether the TST cut-point was 


\begin{tabular}{|c|c|c|c|c|}
\hline & All & HIV-uninfected & HIV-infected & p-value $\#$ \\
\hline Subjects & 253 & 153 & 100 & \\
\hline Age years & $34(18-64)$ & $32(18-64)$ & $35(19-54)$ & NS \\
\hline \multicolumn{5}{|l|}{ Sex } \\
\hline Male & $147(58.1)$ & $100(65.4)$ & $47(47.0)$ & \multirow[t]{2}{*}{0.0043} \\
\hline Female & 106 (41.9) & 53 (34.6) & 53 (53.0) & \\
\hline \multicolumn{5}{|l|}{ Ethnicity } \\
\hline African descent & $200(79.1)$ & $105(68.6)$ & 95 (95.0) & \multirow[t]{2}{*}{$<0.0001$} \\
\hline Other & 53 (20.9) & $48(31.4)$ & $5(5.0)$ & \\
\hline Body mass index $\mathrm{kg} \cdot \mathrm{m}^{-2}$ & $19.8(13.4-43.9)$ & $19.3(13.4-34.5)$ & $20.8(16.0-43.9)$ & NS \\
\hline \multicolumn{5}{|l|}{ BCG status } \\
\hline Vaccinated & $32(12.6)$ & $19(12.4)$ & $13(13.0)$ & NS \\
\hline Not vaccinated & $30(11.9)$ & $18(11.8)$ & 12 (12.0) & NS \\
\hline Unknown & $191(75.5)$ & $116(75.8)$ & 75 (75.0) & NS \\
\hline \multicolumn{5}{|c|}{ Tuberculosis smear status" } \\
\hline+++ & $71(28.1)$ & $58(37.9)$ & $15(15.0)$ & $<0.0001$ \\
\hline++ & $47(18.6)$ & $32(20.9)$ & $15(15.0)$ & NS \\
\hline+ & $46(18.2)$ & 31 (20.3) & $13(13.0)$ & NS \\
\hline Scanty positive $^{+}$ & $38(15.0)$ & $18(11.8)$ & $20(20.0)$ & NS \\
\hline Negative & $49(19.4)$ & $14(9.2)$ & 35 (35.0) & $<0.0001$ \\
\hline
\end{tabular}

Data are presented as $\mathrm{n}$, median (range) or $\mathrm{n}(\%)$, unless otherwise stated. BCG: bacille Calmette-Guerin; NS: nonsignificant. \#: comparisons between HIV subgroups (Fisher's exact test); ^: two missing results; ${ }^{+}:<10$ acid-fast bacilli per 100 high-power fields.

differentiated at $15 \mathrm{~mm}$ for HIV-uninfected and $5 \mathrm{~mm}$ for HIV-infected $(\mathrm{p}<0.0001$ and $\mathrm{p}=0.0002)$ or kept constant at $10 \mathrm{~mm}$ for all $(\mathrm{p}<0.0001$ and $\mathrm{p}<0.0001$; online supplementary table S6).

Combining C-Tb and QFT-GIT results increased the sensitivity significantly from $73.9 \%$ in $\mathrm{C}$ - $\mathrm{Tb}$ alone to 83.4\% when combined with QFT-GIT $(\mathrm{p}<0.0001)$ in all participants, from $76.7 \%$ to $91.1 \%(\mathrm{p}<0.0001)$ in HIV-uninfected and from $69.5 \%$ to $81.1 \%(\mathrm{p}<0.0026)$ in HIV-infected (table 3$)$.

\section{Effect of low CD4 counts in HIV-infected participants}

We evaluated the influence of low CD4 T-cell counts on the magnitude of C-Tb, TST and QFT-GIT responses by grouping all HIV-infected participants into subgroups with decreasing CD4 counts (figure 4). All three tests showed a trend for decreasing magnitudes of responses (induration size or interferon- $\gamma$ release) with decreasing CD4 count (figure $4 \mathrm{a}-\mathrm{c}$ ). Compared with the response magnitude of the HIV-uninfected trial population, this decrease was significant for CD4 counts $<100$ cells $\cdot \mathrm{mm}^{-3}$ for C-Tb and $<200$ cells $\cdot \mathrm{mm}^{-3}$ for TST.

To assess the clinical impact of the decreases, we calculated test sensitivities for each CD4 count interval for the three tests (figure $4 \mathrm{~d}-\mathrm{f}$ ). Compared with the HIV-uninfected trial population, sensitivity was significantly reduced only for CD4 counts $<100$ cells $\cdot \mathrm{mm}^{-3}$ for all three tests $(\mathrm{p}=0.0029, \mathrm{p}=0.0001$ and $\mathrm{p}=0.0139$ for $\mathrm{C}-\mathrm{Tb}$, TST and QFT-GIT, respectively).

\section{Safety of C-Tb and TST}

The majority of all adverse events in the FAS were injection-site reactions (table 4 and online supplementary table S7). For C-Tb, 228 reactions were reported, of which $81 \%$ were rated as mild, $15 \%$ as moderate and $4 \%$ as severe. For TST, 276 reactions were reported, of which $83 \%$ were mild, $15 \%$ moderate and $3 \%$ severe. The most common injection-site reaction was pruritus. Significantly fewer participants experienced at least one adverse injection-site reaction due to $\mathrm{C}$-Tb than due to TST (47.4\% versus $59.3 \%$; $\mathrm{p}=0.0003)$. Among HIV-infected participants, 44/110 (44.0\%) experienced an adverse injection-site reaction to $\mathrm{C}-\mathrm{Tb}$ compared with $76 / 153(49.7 \%)$ of the HIV-uninfected participants ( $\mathrm{p}=0.440)$. For TST, the proportions were 53/100 (53.0\%) compared with 97/153 (63.4\%) ( $\mathrm{p}=0.117)$.

As the trial design included concomitant administration of C-Tb and TST, it was not possible to distinguish whether a systemic event was related to C-Tb or TST, or possibly both. During the trial, $36(14 \%)$ participants experienced a related systemic adverse event as assessed by the site investigators. The most frequently reported related systemic adverse events were headache $(8.7 \%)$ and procedural pain (1.6\%). The majority of these were rated as mild. There was no observed difference between HIV-infected 

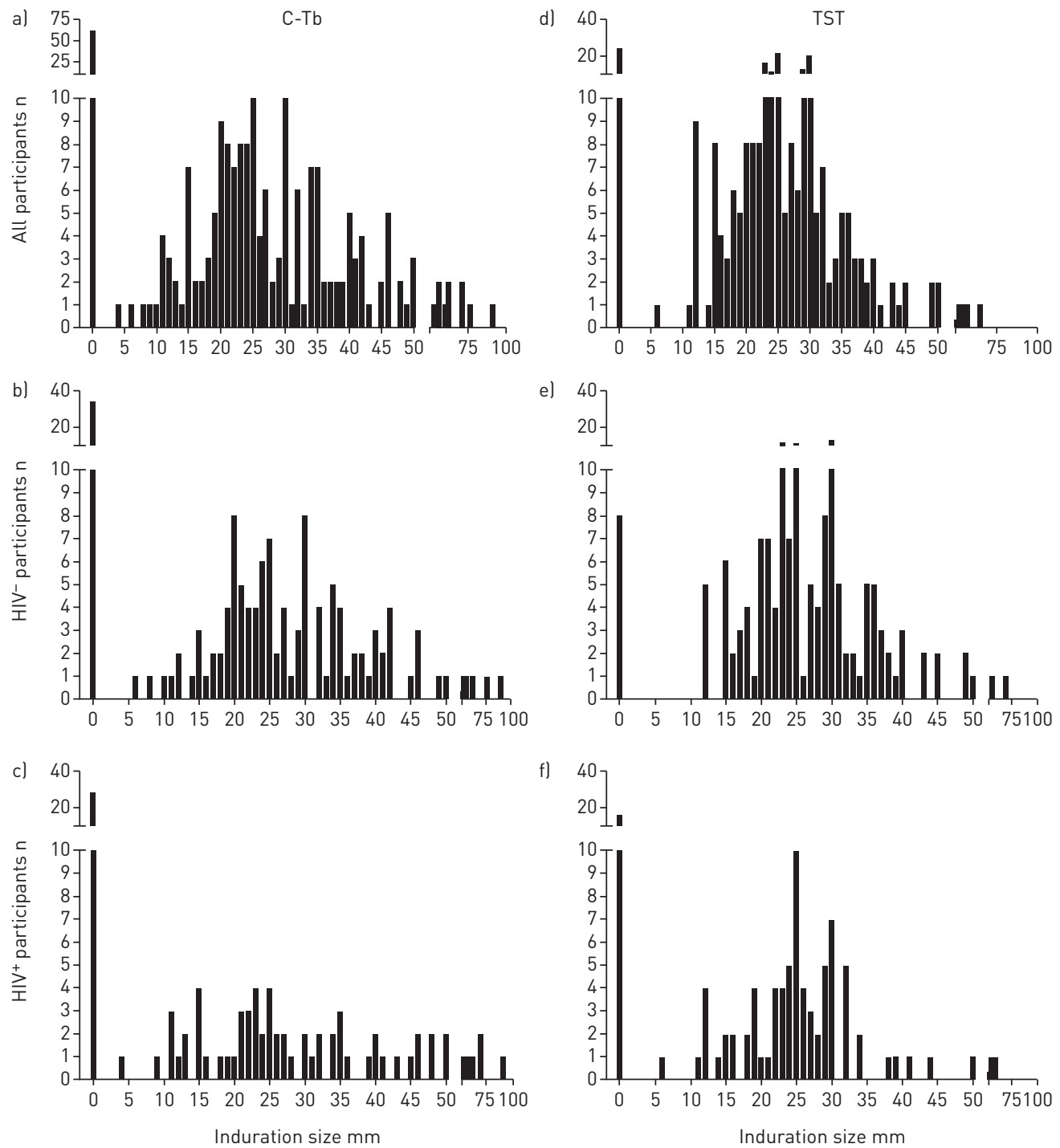

FIGURE $2 \mathrm{a}-\mathrm{c}$ ) C-Tb and $d-f$ ) tuberculin skin test (TST) induration size for $a, d$ ) all participants in the per protocol population ( $n=241$ ), b, e) HIV-uninfected participants ( $n=146$ ) and c, f) HIV-infected participants ( $n=95)$. Each black bar represents the number of participants with a given induration size. Note x-axis split at $50 \mathrm{~mm}$.

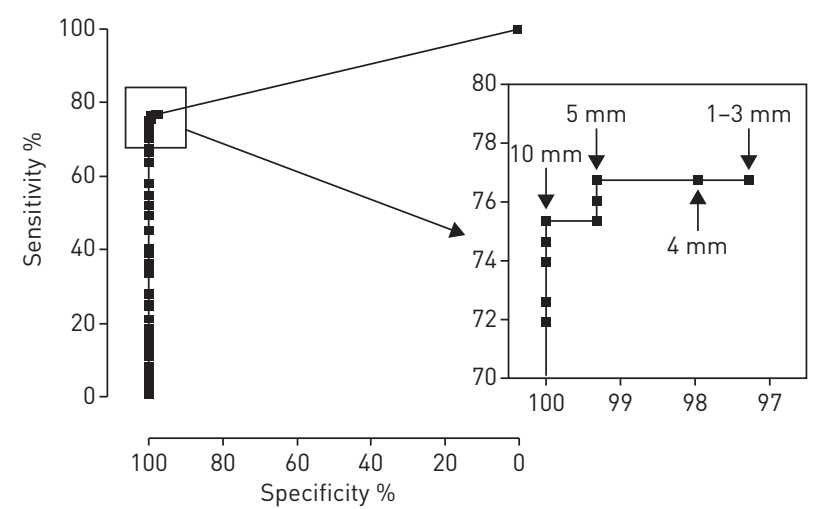

FIGURE 3 C-Tb receiver operating characteristic (ROC) curve showing the relationship between sensitivity and accompanying specificity dependent on test cut-point. Each black square represents the sensitivity and specificity at a given cut-point from 0 to $65 \mathrm{~mm}$. The ROC curve is based on data from 146 HIV-uninfected participants in the per protocol population and $147 \mathrm{HIV-uninfected} \mathrm{bacille} \mathrm{Calmette-Guerin-vaccinated} \mathrm{healthy}$ controls from the previously published specificity trial [13] (online supplementary figure S6). A portion of the curve is magnified for clarification. The optimal cut-point was $5 \mathrm{~mm}$. 
TABLE 2 Sensitivity of C-Tb, tuberculin skin test (TST) and QuantiFERON-TB Gold In-Tube (QFT-GIT)

\begin{tabular}{lcccc} 
& Cut-point & Positive result & Sensitivity & p-value $^{\#}$ \\
\hline All $\mathbf{n = 2 4 1}$ & & & & \\
C-Tb & $\geqslant 5 \mathrm{~mm}$ & 178 & $73.9(67.8-79.3)$ & \\
TST & All $\geqslant 10 \mathrm{~mm}$ & 216 & $89.6(85.1-93.2)$ & 0.0001 \\
TST & $\mathrm{HIV}^{-} \geqslant 15 \mathrm{~mm} / \mathrm{HIV}^{+} \geqslant 5 \mathrm{~mm}$ & 212 & $88.0(83.2-91.5)$ & 0.0001 \\
QFT-GIT & $\geqslant 0.35 \mathrm{IU} \cdot \mathrm{mL}^{-1}$ & 181 & $75.1(69.3-80.2)$ & 0.030 \\
HIV-uninfected $\mathbf{n = 1 4 6}$ & & & & \\
C-Tb & $\geqslant 5 \mathrm{~mm}$ & 112 & $76.7(69.0-83.3)$ & \\
TST & $\geqslant 15 \mathrm{~mm}$ & 133 & $91.1(85.3-95.2)$ & 0.0002 \\
TST & $\geqslant 10 \mathrm{~mm}$ & 138 & $94.5(89.5-97.6)$ & 0.0001 \\
QFT-GIT & $\geqslant 0.35 \mathrm{IU} \cdot \mathrm{mL}^{-1}$ & 112 & $76.7(69.2-82.9)$ & 0.164 \\
HIV-infected $\mathbf{n = 9 5}$ & $\geqslant 5 \mathrm{~mm}$ & & & \\
C-Tb & $\geqslant 5 \mathrm{~mm}$ & 76 & $69.5(59.2-78.5)$ & \\
TST & $\geqslant 10 \mathrm{~mm}$ & 78 & $82.1(72.9-89.2)$ & 0.006 \\
TST & $\geqslant 0.35 \mathrm{IU} \cdot \mathrm{mL}^{-1}$ & 69 & $72.6(62.9-80.6)$ & 0.121 \\
QFT-GIT & & 79.12 & \\
\hline
\end{tabular}

Data are presented as $\mathrm{n}$ or $\%(95 \% \mathrm{CI})$, unless otherwise stated. Based on the per protocol population. \#: McNemar's test compared with C-Tb. Participants with indeterminate QFT-GIT results were excluded from McNemar's test.

and HIV-uninfected participants. The majority of participants (TB patients) had haematology parameters out of normal range at inclusion, but the percentage of out-of-range values had decreased 28 days after skin test injections. There were five serious adverse events reported during the trial (obstructive jaundice, drug induced hepatitis, right sided hydrothorax, decreased CD4 T-cell count and hyperglycaemia). All were assessed as not related to the C-Tb skin test.

\section{Discussion}

Here, for the first time to the best of our knowledge, we report the sensitivity of C-Tb derived from a double-blinded randomised clinical trial that enrolled $253 \mathrm{~TB}$ patients, of which 100 were HIV-infected. Using ROC curve analysis we found the optimal cut-point to be $\geqslant 5 \mathrm{~mm}$. At this cut-point the $\mathrm{C}-\mathrm{Tb}$ sensitivity was $73.9 \%$, which was similar to QFT-GIT (75.1\%), although discordant results between the two tests were seen in $22 \%$ of participants. Although TST sensitivity was higher than both C-Tb and QFT-GIT in this trial, the major concern is lack of specificity particularly in BCG-vaccinated individuals. C-Tb sensitivity was only significantly diminished in participants with HIV infection if CD4 counts were $<100$ cells $\cdot \mathrm{mm}^{-3}$.

As C-Tb and QFT-GIT share a broadly similar antigenic profile, a similar sensitivity was expected. However, C-Tb detected 29 patients deemed negative or indeterminate by QFT-GIT, and QFT-GIT detected 32 patients deemed negative by $\mathrm{C}-\mathrm{Tb}$. The discordance presumably reflects the slightly different antigenic profiles (TB 7.7 in QFT-GIT), fundamentally different immunological pathways that are being measured (complex skin test reaction versus single cytokine in vitro assay), different biological readouts and different repertoires of T-cells being interrogated (circulating versus specialised skin-homing T-cells),

TABLE 3 Combined sensitivity of C-Tb and QuantiFERON-TB Gold In-Tube (QFT-GIT)

\begin{tabular}{lcccc} 
& \multicolumn{3}{c}{ Sensitivity (TESEC-04) } & Specificity (TESEC-03) ${ }^{\#}$ \\
\cline { 2 - 4 } & All & HIV-uninfected & HIV-infected & \\
\hline Subjects & 241 & 146 & 95 & 151 \\
C-Tb & $73.9(67.8-79.3)$ & $76.7(69.0-83.3)$ & $69.5(59.2-78.5)$ & $99.3(96.0-100)$ \\
QFT-GIT & $75.1(69.3-80.2)$ & $76.7(69.2-82.9)$ & $72.6(62.9-80.6)$ & $100(97.0-100)$ \\
Combined & $83.4(78.2-87.6)$ & $91.1(82.3-94.8)$ & $81.1(71.9-87.8)$ & $99.3(96.0-100)$ \\
p-value & $<0.0001$ & $<0.0001$ & $<0.0026$ & 1 \\
& & & &
\end{tabular}

Data are presented as $\mathrm{n}$ or $\%(95 \% \mathrm{Cl})$, unless otherwise stated. \#: all TESEC-03 participants were HIV-uninfected and QFT-GIT-negative as per inclusion criteria [13]; ๆ: McNemar's test: C-Tb and QFT-GIT combined versus $\mathrm{C}$ - $\mathrm{Tb}$ alone. 
$\mathrm{C}-\mathrm{Tb}$
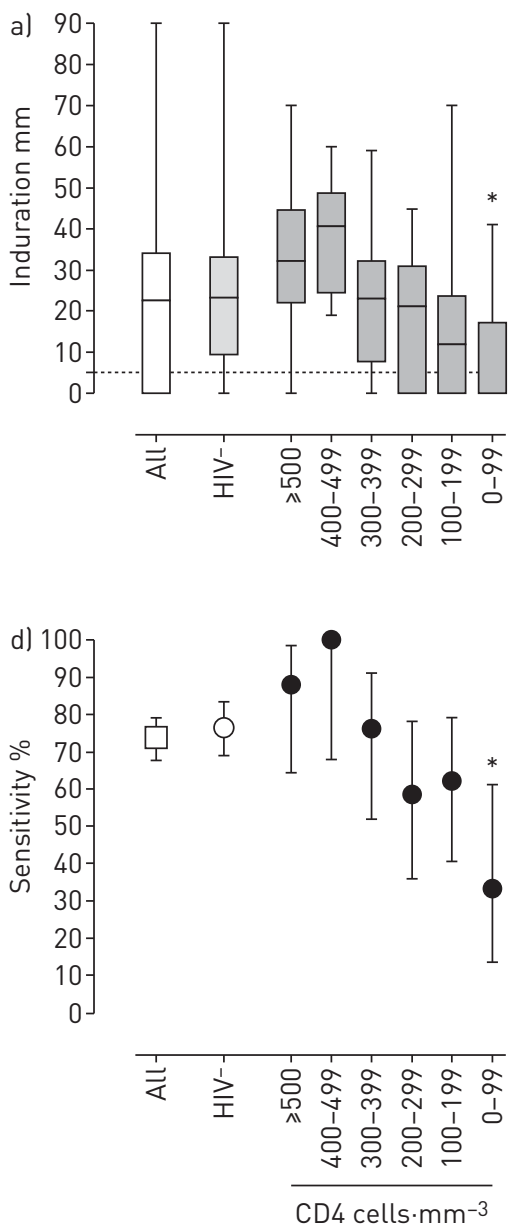

TST
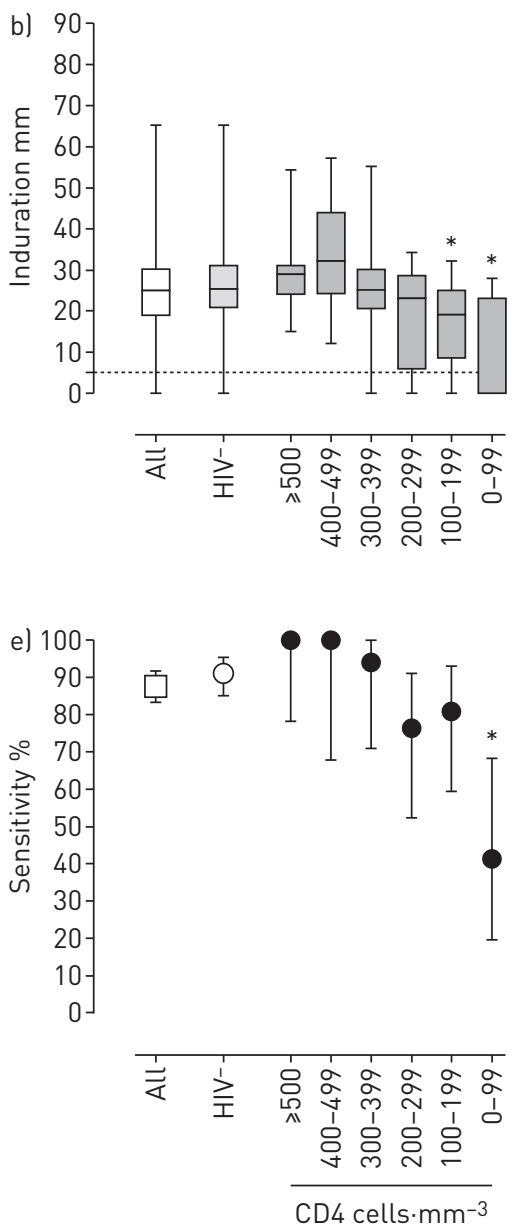

QFT-GIT
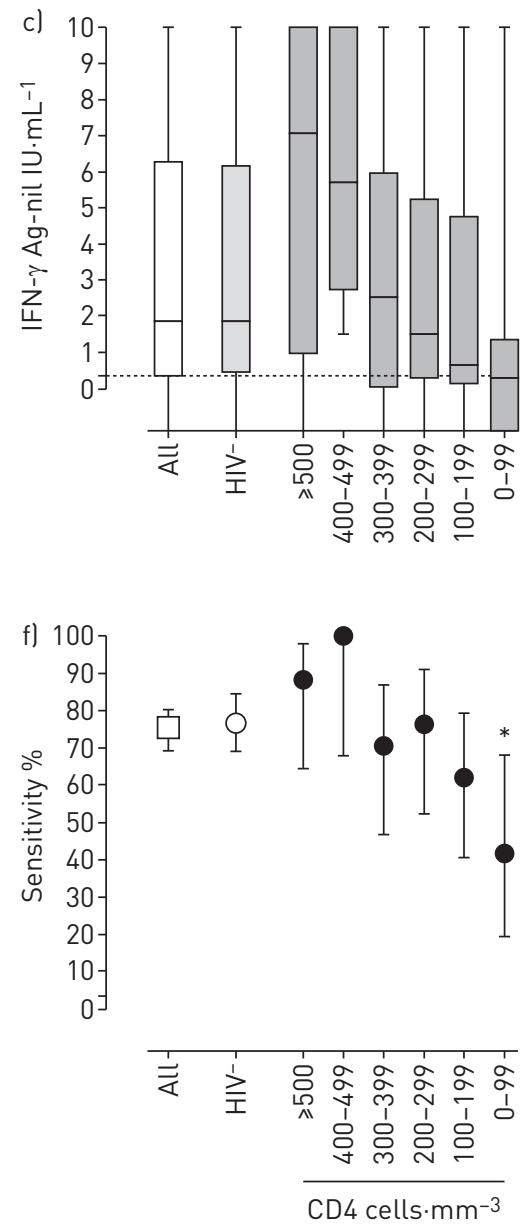

FIGURE $4 \mathrm{a}-\mathrm{c}$ ) Box-and-whisker plots showing the magnitude of immune responses for all participants (All, $\mathrm{n}=240$ ), HIV-uninfected participants (HIV $\left.{ }^{-}, n=146\right)$ and HIV-infected participants split into six subgroups according to CD4 cell counts $(\geqslant 500, n=12 ; 400-499, n=21 ; 300-399, n=17 ; 200-$ 299, $n=17 ; 100-199, n=10 ; 0-99, n=17)$. One participant had a CD4 count missing. Whiskers indicate minimum and maximum values. $d-f)$ Line plots showing sensitivity $(95 \% \mathrm{CI})$ for all participants, HIV-uninfected participants and HIV-infected CD4 count subgroups. TST: tuberculin skin test; QFT-GIT: QuantiFERON-TB Gold In-Tube; IFN: interferon; Ag: antigen. Each CD4 count subgroup was compared with the HIV-uninfected group. *: $p<0.05$.

\section{TABLE 4 Adverse injection-site reactions in 253 patients with active tuberculosis}

\section{C-Tb}

Total adverse reactions reported

Participants with at least one adverse injection-site reaction (any type)

Participants experiencing

Pruritus

Pain

Swelling

Vesicles

Rash

Inflammation

Ulceration
Erythema

$228(45.2)$
$120(47.4)$
$88(34.8)$
$43(17.0)$
$42(16.6)$
$38(15.0)$
$11(4.4)$
$2(0.8)$
$2(0.8)$
$1(0.4)$

$228(45.2)$

88 (34.8)

$2(16.6)$

$38(15.0)$

$2(0.8)$

$1(0.4)$
TST

Difference $p$-value ${ }^{\#}$

Data are presented as $\mathrm{n}(\%)$, unless otherwise stated. TST: tuberculin skin test. ${ }^{\#}$ : McNemar's test.
276 (54.8)

$150(59.3)$

0.0003

$109(43.1)$

0.0104

$52(20.6)$

0.1237

0.7194

0.8445

0.0269

0.0412

1.0000

1.0000 
amongst others $[18,19]$. One obvious difference is the longer time span during which T-cells are exposed to the antigens with the skin test (up to $72 \mathrm{~h}$ compared with $16-24 \mathrm{~h}$ ), thus potentially reflecting central and effector memory T-cell readouts. Genetic factors may also influence whether skin test induration occurs and its relative magnitude [20]. In contrast, TST sensitivity was $\sim 89 \%$. Although this appears to be higher than reported in other studies [21], it is in agreement with previous studies from South Africa [22]. The latter is likely due to the high force of infection [23], ongoing T-cell priming by the high prevalence of environmental mycobacteria [24] and previous BCG vaccination [9].

Stratifying participants by HIV status and CD4 counts, we observed a trend towards lower magnitudes of responses and lower test sensitivities for C-Tb, TST and QFT-GIT at lower CD4 counts. This is a well-known effect of HIV-induced immunosuppression [25-28]. A drop in the magnitude of responses was evident in patients with CD4 counts $<400$ cells $\cdot \mathrm{mm}^{-3}$. For C-Tb as well as for the comparators, this reached significance only in the lowest CD4 count interval $\left(0-99\right.$ cells $\left.\cdot \mathrm{mm}^{-3}\right)$. We presume this is due to the attenuated ability to mount an inflammatory skin test response.

Current recommendations suggest lowering the TST cut-point to $5 \mathrm{~mm}$ in HIV-infected individuals [4], as done in the present work. At this cut-point the TST sensitivity was significantly and better than both QFT-GIT and C-Tb, which is in line with that seen in other studies [29]. In contrast, lowering the C-Tb cut-point from 5 to $1 \mathrm{~mm}$ in $\mathrm{HIV}$-infected individuals did not improve test performance. Notably, a C-Tb $5 \mathrm{~mm}$ cut-point showed a superior specificity in BCG-vaccinated healthy volunteers compared with TST [13]. Importantly, these data collectively suggest that $\mathrm{C}-\mathrm{Tb}$, in contrast to TST, can be interpreted using a single universal cut-point, which will improve ease of use and potentially reduce the number of false-negative responders with unknown HIV status. Indeed, maximal sensitivity is desired when testing high-risk patients, including HIV-infected individuals, children and those on immune-suppressive therapy, e.g. anti-tumour necrosis factor- $\alpha$ treatment [9]. Thus, in day-to-day use where maximal sensitivity is crucial, adding QFT-GIT testing, if C-Tb is negative, would seem sensible, but needs to be explored further in appropriately designed studies.

$\mathrm{C}$ - Tb was well tolerated in the trial population of HIV-infected and HIV-uninfected TB patients. The majority of adverse events were injection-site reactions, but at a significantly reduced frequency compared with PPD-driven TST. As the trial design included concomitant administration of C-Tb and TST, it was not possible to distinguish whether a specific event was related to C-Tb or TST, or both. This was of little consequence, as the number of related systemic adverse events was small and adverse events were in general mild. It is worth noting that many blood parameters were abnormal at inclusion, reflecting the diseased state of the recruited population. Indeed, they improved during the course of treatment.

There are several limitations to our findings. The sensitivity estimate reported here is determined from the same data from which the cut-off was identified. Thus, additional validation studies in different settings are needed to confirm the sensitivity reported here. The sensitivity estimates for active TB may not be similar to that of LTBI and are likely to be lower as TB is an immune-suppressive condition. However, as there is no reliable diagnostic standard for LTBI, active TB was used as a comparable surrogate. We did not perform or test alternative proxy measures of performance, such as including close contacts to correlate test results with exposure gradients and evaluate the predictive value for active TB. Both QFT-GIT and TST are known to have poor predictive value for progression to TB disease. As C-Tb shares the same antigens as QFT-GIT, this novel skin test is not expected to be a major advance in this respect. Compared with QFT-GIT, the main advantages of $\mathrm{C}-\mathrm{Tb}$ are expected to be simplicity, as $\mathrm{C}$ - $\mathrm{Tb}$ requires no blood draw, no handling and transport of blood samples, and no specialised laboratory equipment. However, QFT-GIT has the advantage of having inbuilt negative and positive control tubes not present in a skin test format. A future trial will report on $\mathrm{C}$ - $\mathrm{Tb}$ performance in a contact-tracing design, thus correlating $\mathrm{C}$ - $\mathrm{Tb}$ positivity rates with an exposure gradient. A phase 3 trial evaluating $\mathrm{C}-\mathrm{Tb}$ in $\mathrm{TB}$ suspects is in the process of being concluded.

In conclusion, this first assessment of $\mathrm{C}-\mathrm{Tb}$ in active $\mathrm{TB}$ shows similar sensitivity compared with QFT-GIT. In contrast to TST, C-Tb can be used with a single universal cut-point regardless of BCG and HIV status. Further studies will validate if $\mathrm{C}-\mathrm{Tb}$ may be a valuable alternative to, or replacement for, $\mathrm{PPD}$ TST and/or QFT-GIT.

\section{Acknowledgements}

We thank all investigators, staff and trial participants at all trial sites. We thank the members of the Data Safety Monitoring Board: Dr Pernille Ravn, Dr Luca Richeldi and Dr Christian Lienhardt.

\section{References}

1 Dheda K, Barry C III, Maartens G. Tuberculosis. Lancet 2015 [in press; DOI: 10.1016/S0140-6736(15)00151-8]. 
Esmail H, Barry CE, Young DB, et al. The ongoing challenge of latent tuberculosis. Philos Trans R Soc Lond B Biol Sci 2014; 369: 20130437.

3 Mack U, Migliori GB, Sester M, et al. LTBI: latent tuberculosis infection or lasting immune responses to M. tuberculosis? A TBNET consensus statement. Eur Respir J 2009; 33: 956-973.

4 Dheda K, Smit R, van Z, et al. T-cell interferon- $\gamma$ release assays for the rapid immunodiagnosis of tuberculosis: clinical utility in high-burden vs. low-burden settings. Curr Opin Pulm Med 2009; 15: 188-200.

5 Farhat M, Greenaway C, Pai M, et al. False-positive tuberculin skin tests: what is the absolute effect of BCG and non-tuberculous mycobacteria? Int J Tuberc Lung Dis 2006; 10: 1192-1204.

6 Brock I, Munk ME, Kok-Jensen A, et al. Performance of whole blood IFN- $\gamma$ test for tuberculosis diagnosis based on PPD or the specific antigens ESAT-6 and CFP-10. Int J Tuberc Lung Dis 2001; 5: 462-467.

7 Johnson PD, Stuart RL, Grayson ML, et al. Tuberculin-purified protein derivative-, MPT-64-, and ESAT-6-stimulated gamma interferon responses in medical students before and after Mycobacterium bovis BCG vaccination and in patients with tuberculosis. Clin Diagn Lab Immunol 1999; 6: 934-937.

8 Mori T, Sakatani M, Yamagishi F, et al. Specific detection of tuberculosis infection. Am J Respir Crit Care Med 2004; 170: 59-64.

9 Pai M, Denkinger CM, Kik SV, et al. Gamma interferon release assays for detection of Mycobacterium tuberculosis infection. Clin Microbiol Rev 2014; 27: 3-20.

10 Adams S, Ehrlich R, Baatjies R, et al. Incidence of occupational latent tuberculosis infection in South African healthcare workers. Eur Respir J 2015; 45: 1364-1373.

11 Dheda K, Ruhwald M, Theron G, et al. Point-of-care diagnosis of tuberculosis: past, present and future. Respirology 2013; 18: 217-232.

12 Bergstedt W, Tingskov PN, Thierry-Carstensen B, et al. First-in-man open clinical trial of a combined rdESAT-6 and rCFP-10 tuberculosis specific skin test reagent. PLoS One 2010; 5: e11277.

13 Aggerbeck H, Giemza R, Joshi P, et al. Randomised clinical trial investigating the specificity of a novel skin test (C-Tb) for diagnosis of M. tuberculosis infection. PLoS One 2013; 8: e64215.

14 Wang G-Z. Efficacy and safety of recombinant Mycobacterium tuberculosis ESAT-6 protein for diagnosis of pulmonary tuberculosis: a phase II trial. Med Sci Monit 2013; 19: 969-977.

15 Kiselev VI, Baranovskiı̌ PM, Rudykh IV, et al. [Clinical trials of the new skin test Diaskintest for the diagnosis of tuberculosis]. Probl Tuberk Bolezn Legk 2009; 2: 11-16.

16 Schulz KF, Altman DG, Moher D CONSORT 2010 statement: updated guidelines for reporting parallel group randomised trials. PLoS Med 2010; 7: e1000251.

17 Aggerbeck H, Madsen SM. Safety of ESAT-6. Tuberculosis 2006; 86: 363-373.

18 Andersen P, Munk M, Pollock J, et al. Specific immune-based diagnosis of tuberculosis. Lancet 2000; 356: 1099-1104.

19 Gallant CJ, Cobat A, Simkin L, et al. Tuberculin skin test and in vitro assays provide complementary measures of antimycobacterial immunity in children and adolescents. Chest 2010; 137: 1071-1077.

20 Cobat A, Gallant CJ, Simkin L, et al. Two loci control tuberculin skin test reactivity in an area hyperendemic for tuberculosis. J Exp Med 2009; 206: 2583-2591.

21 Metcalfe JZ, Everett CK, Steingart KR, et al. Interferon- $\gamma$ release assays for active pulmonary tuberculosis diagnosis in adults in low- and middle-income countries: systematic review and meta-analysis. J Infect Dis 2011; 204: S1120-S1129.

22 Tsiouris SJ, Coetzee D, Toro PL, et al. Sensitivity analysis and potential uses of a novel gamma interferon release assay for diagnosis of tuberculosis. J Clin Microbiol 2006; 44: 2844-2850.

23 Mahomed H, Ehrlich R, Hawkridge T, et al. TB incidence in an adolescent cohort in South Africa. PLoS One 2013; 8: e59652.

24 Botha L, Gey van Pittius NC, van Helden PD. Mycobacteria and disease in Southern Africa. Transbound Emerg Dis 2013; 60: 147-156.

25 Cattamanchi A, Smith R, Steingart KR, et al. Interferon-gamma release assays for the diagnosis of latent tuberculosis infection in HIV-infected individuals: a systematic review and meta-analysis. J Acquir Immune Defic Syndr 2011; 56: 230-238.

26 Elzi L, Schlegel M, Weber R, et al. Reducing tuberculosis incidence by tuberculin skin testing, preventive treatment, and antiretroviral therapy in an area of low tuberculosis transmission. Clin Infect Dis 2007; 44: 94-102.

27 Aabye MG, Ravn P, PrayGod G, et al. The impact of HIV infection and CD4 cell count on the performance of an interferon gamma release assay in patients with pulmonary tuberculosis. PLoS One 2009; 4: e4220.

28 Dheda K, Lalvani A, Miller R, et al. Performance of a T-cell-based diagnostic test for tuberculosis infection in HIV-infected individuals is independent of CD4 cell count. Aids 2005; 19: 2038-2041.

29 Cobelens FG, Egwaga SM, Ginkel T, et al. Tuberculin skin testing in patients with HIV infection: limited benefit of reduced cutoff values. Clin Infect Dis 2006; 43: 634-639. 